\title{
Increased ICP and its cerebral haemodynamic sequelae
}

Joseph Donnelly $^{1 *}$, Marek Czosnyka ${ }^{1,2}$, Spencer Harland ${ }^{3}$, Georgios V. Varsos ${ }^{1}$, Danilo Cardim ${ }^{1}$, Chiara Robba ${ }^{1}$, Xiuyun Liu ${ }^{1}$, Philip N. Ainslie ${ }^{4}$, Peter Smielewski ${ }^{1}$,

${ }^{1}$ Brain Physics Laboratory, Division of Neurosurgery, Department of Clinical Neurosciences, Cambridge Biomedical Campus, University of Cambridge, Cambridge, UK

${ }^{2}$ Institute of Electronic Systems, Warsaw University of Technology, Warsaw, Poland

${ }^{3}$ Queen Elizabeth Hospital, Birmingham, UK

${ }^{4}$ University of British Columbia, Canada

Financial support for this project: No specific funding for this study

Key words: Intracranial pressure, cerebral haemodynamics, autoregulation, cerebral perfusion pressure

Corresponding author: Joseph Donnelly

Brain Physics Laboratory, Division of Neurosurgery, Department of Clinical Neurosciences, Cambridge Biomedical Campus, University of Cambridge, Cambridge CB2 0QQ, UK

Fax: +44 (0) 1223 216926, Tel: +44 (0) 1223 336946, e-mail: jd634@cam.ac.uk 


\begin{abstract}
Objectives: Increased intracranial pressure (ICP) is a pathologic feature of many neurological diseases, however the local and systemic sequelae of raised ICP are incompletely understood. Using an experimental paradigm, we aimed to describe the cerebrovascular consequences of acute increases in ICP
\end{abstract}

Materials and methods: We assessed cerebral haemodynamics (mean arterial blood pressure-MAP, ICP, Laser doppler flowmetry-LDF, basilar artery Doppler flow velocity-Fv and estimated vascular wall tension-WT) in 27 basilar artery-dependent rabbits during experimental (artificial lumbar CSF infusion) intracranial hypertension. Wall tension (WT) was estimated as the difference between critical closing pressure and ICP.

Results: From baseline $(\sim 9 \mathrm{mmHg})$ to moderate increases in ICP $(\sim 41 \mathrm{mmHg})$, cortical LDF decreased (LDF 100 to $39.1 \% \mathrm{p}<0.001$ ) while mean global $\mathrm{Fv}$ was unchanged ( 47 to $45 \mathrm{~cm} / \mathrm{s} ; \mathrm{P}=$ 0.38). In addition, MAP increased ( 88.8 to $94.2 \mathrm{mmHg}$; $\mathrm{p}<0.01$ and WT decreased (19.3 to 9.8 $\mathrm{mmHg}, \mathrm{p}<0.001)$. From moderate to high ICP $(\sim 75 \mathrm{mmHg})$, both global Fv and cortical LDF decreased (Fv 45 to $31.3 \mathrm{~cm} / \mathrm{s} \mathrm{p}<0.001$, LDF 39.1 to 13.3\%, p <0.001) while MAP increased further (94.2 to $114.5 \mathrm{mmHg} ; \mathrm{p}<0.001)$ and estimated WT was unchanged $(9.7$ to $9.6 \mathrm{mmHg}$; $\mathrm{p}=0.35$ ).

Conclusion: In this analysis, we demonstrate a cortical vulnerability to increases in ICP and two ICP dependent cerebroprotective mechanisms: with moderate increases in ICP, WT decreases and MAP increases to buffer cerebral perfusion, while with severe increases of ICP, an increase MAP predominates. 


\section{Introduction}

Due to the rigid skull incasing the cerebrum, increased intracranial volume from whatever cause can lead to raised intracranial pressure (ICP). The consequences of a raised intracranial pressure are universally harmful and include impaired cerebral blood flow (CBF), electrical activity, and metabolism. Therefore, the avoidance of raised ICP is pivotal in the management of many neurological conditions where acute changes in cerebral volume are possible including traumatic brain injury, subarachnoid haemorrhage, or acute hydrocephalus [1].

Increases in ICP lead to a decrease in cerebral perfusion pressure (CPP; calculated as mean arterial pressure -ICP) and thus can limit perfusion to the brain [2]. The brain however has an intrinsic mechanism to protect itself from injury due to low CPP; cerebral autoregulation [3]. While the haemodynamic response to decreases in MAP have been well described, the response to increases in intracranial pressure are less well understood.

In this study, we revisited the question of how raised ICP affects cerebral haemodynamics using a rabbit model of experimental intracranial hypertension. This is a short summary of unpublished data from experimental material, based upon we recently published a full paper [4].

\section{Methods}

\section{Animals and ethics}

These experiments were carried out in 1995 and 1996 in accordance with the standards provided by the UK Animals Scientific Procedures act of 1986 under a UK home office license and with permission from the institutional animal care and use committee at Cambridge University.

Physiological recordings from lumbar CSF infusions in $28 \mathrm{NZ}$ white rabbits (7 female, 21 male; weight $2.7-3.7 \mathrm{~kg}$ ) were retrospectively analysed [5]. The experimental procedures for this specific experiment has been described in previous publications [6]. Briefly, ICP was monitored using an intraparenchymal microsensor (Codman and Shurtleff, Raynham, MA, USA), cortical blood flow 
with a laser Doppler flowmetry probe (Moor Instruments, Axbridge, Devon, UK). Basilar artery flow velocity using an $8 \mathrm{MHz}$ Doppler ultrasound probe (PCDop 842, SciMed, Bristol, UK), and ABP using a catheter in the dorsal aorta (GaelTec, Dunvegan, UK). A lumbar laminectomy facilitated insertion of a catheter for the controlled infusion of artificial cerebrospinal fluid. The animals were supported in the Sphinx position using a purpose-built head frame with three-point skull fixation. Ventilation was controlled according to arterial $\mathrm{PCO}_{2}$ via periodic arterial blood gas analyses. All experiments were performed in an animal laboratory at the same time of day.

Importantly these rabbits had their common carotid arteries ligated 2 weeks prior to experimentation so that blood flow to the brain was basilar artery dependent. This made a doppler assessment of global blood flow possible through the insonation of only the basilar artery.

\section{Protocol}

Following 20 minutes of rest, ICP was artificially increased by infusion of Hartmanns solution into the lumbar CSF space. Infusion rates were initially $0.1 \mathrm{~mL} / \mathrm{min}$. ICP increased to reach a plateau of around $40 \mathrm{~mm} \mathrm{Hg}$ after approximately 10 minutes, thereafter the infusion rate was increased to rates between 0.2 and $2 \mathrm{ml} / \mathrm{min}$ to produce severe intracranial hypertension. ICP was increased until the point where diastolic flow velocity approached zero, which corresponded to an ICP of between 60 and $100 \mathrm{~mm} \mathrm{Hg}$ (mean $75 \mathrm{~mm} \mathrm{Hg}$ ) at the termination of the experiment. Rabbits were euthanized with thiopental at the conclusion of the test.

\section{Data acquisition and analysis}

ABP, ICP, basilar artery flow velocity $(\mathrm{Fv})$ and expired $\mathrm{CO}_{2}$ signals were recorded digitally at a sampling frequency of $50 \mathrm{~Hz}$. Data were subsequently analysed off-line using custom-built, commercially available data analysis software (ICM+; http://www.neurosurg.cam.ac.uk/icmplus). LDF was expressed as a.u. minus the 'biological zero' as measured in the asystolic rabbit at the conclusion of the experiment. $\mathrm{CrCP}$ was calculated based on an impedance based methodology as previously described in [7]. Estimated arterial wall tension (WT) was calculated as CrCP- ICP. 
Pairwise comparisons of haemodynamic parameters between the three different ICP conditions ("low", "moderate", and "high") were performed using Student's t-test. The alpha-value was set at 0.05 and no corrections were made for multiple comparisons. Of the available 28 experiments, one was excluded from analysis due to an inability to increase ICP above $30 \mathrm{~mm} \mathrm{Hg}$ with CSF infusion, leaving 27 rabbits for the final analysis. Statistical analysis was performed using IBM SPSS version 21.0 software (IBM Corp., Armonk, USA).

\section{Results:}

Major statistical descriptive of monitored parameters is given in Table 1. Increases in MAP were observed between baseline and moderate and also between moderate high levels of ICP. As a result of the Cushing vasopressor response, despite an increase in ICP by $35 \mathrm{~mm}$ Hg between the 'moderate' and 'high' ICP conditions, CPP only decreased by $14 \mathrm{~mm} \mathrm{Hg}$. Mean flow velocity of the basilar artery was not significantly changed from baseline to moderate ICP, whereas cortical LDF and diastolic flow velocity decreased. End tidal $\mathrm{CO}_{2}$ remained constant throughout the experiment.

The inter-relationships between changes in ICP, CBF, vascular wall tension, and MAP in all 27 rabbits are shown in figure $1 . \mathrm{CBF}$ appears well maintained with up to $60 \mathrm{~mm} \mathrm{Hg}$ increases in ICP. This maintenance of CBF was contributed by both decreases in wall tension and increases in MAP. Wall tension progressively decreased with increasing ICP particularly during early increases in ICP. At the highest levels of ICP, WT started to increase.

\section{Discussion:}

In this experimental investigation, we identified two intrinsic and mechanistically distinct compensatory adaptations to raised ICP: with moderate increases in ICP, CBF is maintained by decreases in vascular wall tension; whereas at higher levels of ICP, cerebral perfusion is protected by the Cushing vasopressor response that maintains CPP. In addition, regional differences in the control 
of cerebral perfusion were observed with cortical blood flow being more sensitive to an increase in ICP than global blood flow.

In these experiments, global CBF was maintained until ICP had increased by $55 \mathrm{~mm} \mathrm{Hg}$ above baseline ICP (Fig. 1). This maintenance of CBF seemed to be mediated by a decrease in estimated vascular wall tension (Fig 1 and Table 1). Estimated wall tension decreased and reached a plateau during ICP increases between 30 and $60 \mathrm{~mm} \mathrm{Hg.} \mathrm{(Fig.} \mathrm{1,} \mathrm{Table} \mathrm{1).} \mathrm{This} \mathrm{plateau} \mathrm{of} \mathrm{vascular} \mathrm{wall}$ tension (at around 6-8 $\mathrm{mm} \mathrm{Hg}$ ) may represent a condition of maximum vasodilation biologically fixed by the rigid collagen fibres in the tunica adventitia [8]. Further increases in ICP caused a vigorous Cushing response as reflected by reductions in HR and increases in MAP (MAP increasing by $>30$ $\mathrm{mm} \mathrm{Hg}$ in 13 out of 27 rabbits). However, in contrast to our hypothesis, this hypertensive response was also present with moderate ICP increases (in 12 out of 27 individuals, the hypertensive response was observed with increases of ICP of less than $25 \mathrm{~mm} \mathrm{Hg}$ ). This relatively 'early' increase in ICP may indicate that the ICP induced increases in MAP play a protective role in maintaining perfusion rather than merely signifying irreversible neurologic damage. In support of this finding, a similar increase MAP in humans has been observed at even moderate ICP during lumbar CSF infusion studies [9].

Using in-vivo global and cortical CBF measurement during dynamic changes in ICP, we demonstrated that cortical blood flow decreased even with moderate increases in ICP whereas global flow (basilar artery flow velocity) was well maintained until higher levels of ICP (Table 1). A potential explanation for the vulnerability of cortical compared to global CBF could be regional variation in vessel anatomy and compliance or could lie in a differential vascular reactivity of cortical compared with non-cortical brain.

In support of a topographic difference in cerebral vascular reactivity, Horsefield et al demonstrated using MRI an intrinsic difference in the autoregulatory efficiency of the grey matter compared to white matter of healthy humans in response to a transient decrease in CPP [10]. Furthermore, in a group of severe TBI patients, Zweifel et al., found there was a higher correlation between CPP and LDF than between CPP and middle cerebral artery Fv [11]. Such cortical sensitivity to high ICP could 
be a mechanism for diverting blood flow to areas of the brain most crucial for survival; the brainstem nuclei.

\section{Limitations}

The current results reflect the cerebral haemodynamic response to raising ICP through the addition of CSF volume. Thus, the observed haemodynamic response may differ somewhat to those observed clinically in the injured, oedematous brain where changes in volume of the intracranial blood, CSF, or parenchymal compartments are all common. The current study represents a retrospective analysis and therefore some specific details of the surgical procedures (such as the functional integrity of the arterial baroreceptors) cannot be assessed. Application of cortical LDF during an infusion of fluid into the subarachnoid space raises the possibility of LDF probe displacement by the infusion. Finally, bilateral ligation of the common carotid could affect cerebral haemodynamics per se. However, this technique ensured that flow velocity measured at the basilar artery represented a global cerebral perfusion and thus provided a well-controlled model to address our proposed questions.

\section{Conclusion}

Decreased vascular wall tension and increased MAP act to protect cerebral perfusion from increases in ICP, however the relative importance of these mechanisms depends on the prevailing ICP. Furthermore, reductions in cortical blood flow due to increases in ICP may not be detected by global measures of perfusion. Multimodal monitoring including global and local techniques may enhance personalized clinical management in neurocritical disease.

Table and figure legends: 
Table 1 Haemodynamic consequences of raised intracranial pressure

\begin{tabular}{|c|c|c|c|c|c|c|c|c|c|}
\hline & \multicolumn{2}{|c|}{ Baseline } & \multicolumn{2}{|c|}{ Moderate ICP } & \multicolumn{3}{|c|}{ High ICP } & \multirow{3}{*}{$\begin{array}{l}\text { Significance } \\
\text { compared to baseline }\end{array}$} & \multirow{3}{*}{$\begin{array}{l}\text { Significance compared } \\
\text { to moderate ICP }\end{array}$} \\
\hline & & & & & Significance & & & & \\
\hline & Mean & SE & Mean & SE & compared to baseline & Mean & SE & & \\
\hline$\overline{\mathrm{ICP}(\mathrm{mm} \mathrm{Hg})}$ & 9.00 & 1.51 & 40.75 & 2.17 & $* *$ & 75.05 & 3.65 & $* *$ & $* *$ \\
\hline MAP (mm Hg) & 88.84 & 3.29 & 94.15 & 3.21 & $*$ & 114.54 & 3.67 & $* *$ & $* *$ \\
\hline CPP (mm Hg) & 79.71 & 3.46 & 53.08 & 2.77 & $* *$ & 39.64 & 2.16 & $* *$ & $* *$ \\
\hline HR (BPM) & 273.27 & 6.96 & 262.73 & 7.64 & $*$ & 227.21 & 11.13 & $* *$ & $* *$ \\
\hline Global Fv mean $(\mathrm{cm} / \mathrm{s})$ & 47.04 & 2.99 & 44.99 & 2.82 & & 31.33 & 2.45 & $* *$ & $* *$ \\
\hline Global Fv diastolic (cm/s) & 34.17 & 2.76 & 30.49 & 2.58 & $*$ & 15.59 & 1.67 & $* *$ & $* *$ \\
\hline Global Fv systolic (cm/s) & 66.93 & 3.64 & 65.51 & 3.33 & & 50.97 & 2.66 & $* *$ & $* *$ \\
\hline WT (mm Hg) & 19.27 & 1.84 & 9.75 & 1.27 & $* *$ & 9.58 & 1.36 & $* *$ & \\
\hline $\mathrm{CrCP}(\mathrm{mm} \mathrm{Hg})$ & 28.42 & 2.05 & 50.61 & 2.12 & $* *$ & 85.09 & 3.99 & $* *$ & $* *$ \\
\hline Cortical LDF (\%) & 100.00 & 0.00 & 39.10 & 29.82 & $* *$ & 13.27 & 5.19 & $* *$ & $* *$ \\
\hline
\end{tabular}


*ICP-intracranial pressure; MAP- mean arterial pressure; CPP-cerebral perfusion pressure; HR- heart rate; Fv-flow velocity; WT- wall tension; CrCPCritical closing pressure; LDF-Laser Doppler flow 
Global Fv

(\%)

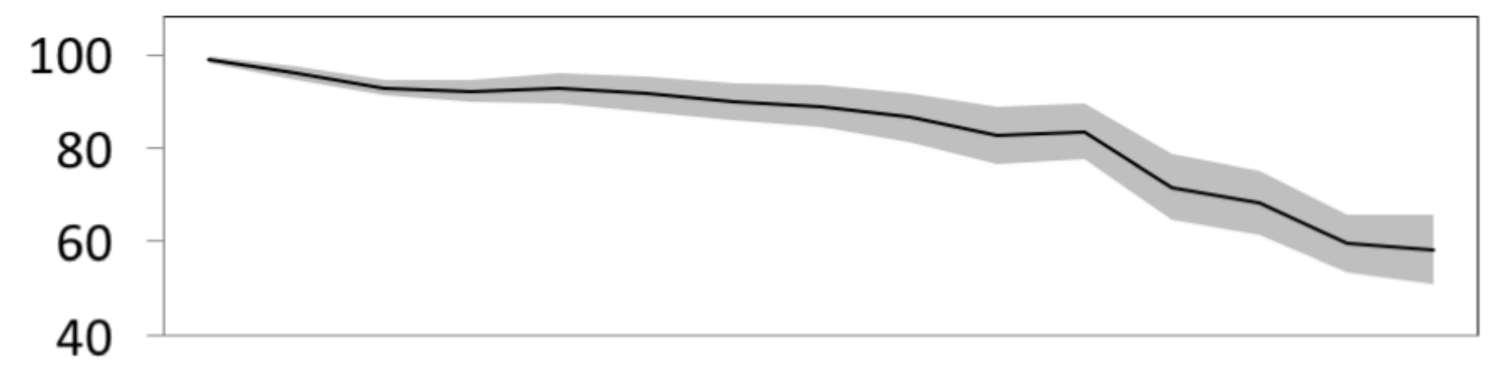

Wall tension (mm Hg)

MAP

(mm Hg)

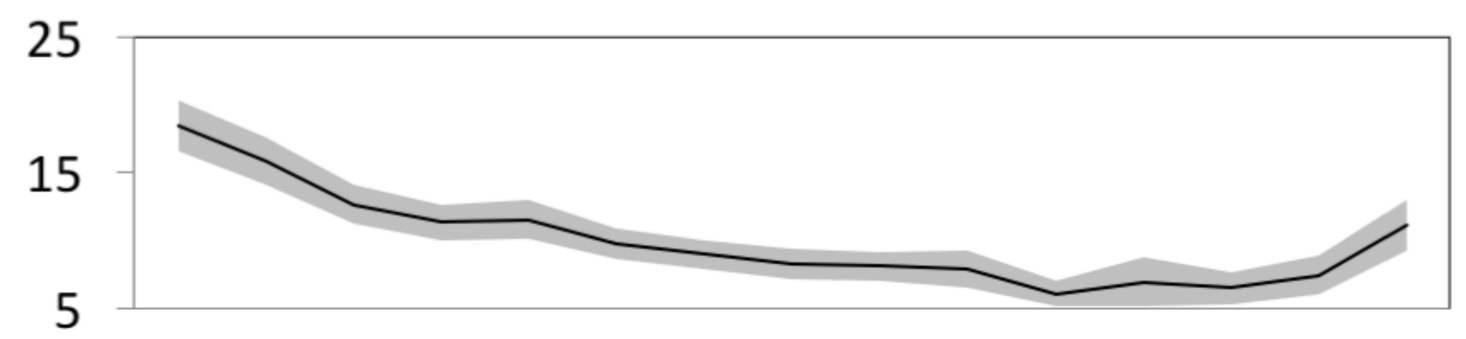

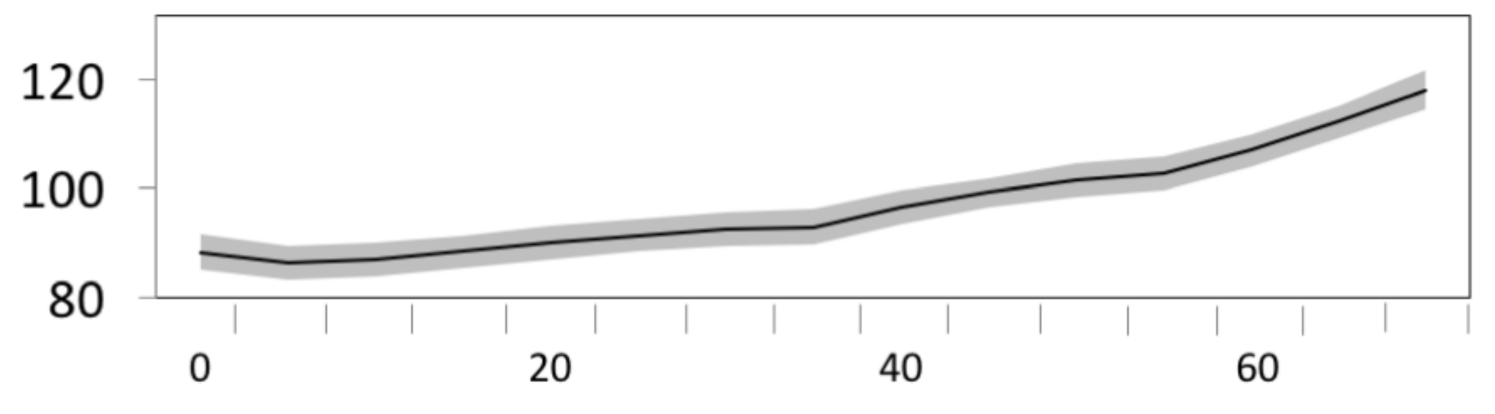

Increase in ICP

(mm Hg) 
Figure 1 The haemodynamic response to increased intracranial pressure induced by infusion of artificial CSF in NZ rabbits (n=27)

With moderate increases in ICP, global CBF (Basilar artery Fv after common carotid ligation) is maintained through a decrease in vascular wall tension. With more severe increases in ICP (greater than $20 \mathrm{~mm} \mathrm{Hg}$ increase in ICP), a cushing response mediated increase in MAP helps to maintain CBF. 
References

1. Donnelly J, Budohoski KP, Smielewski P, Czosnyka M (2016) Regulation of the cerebral circulation: bedside assessment and clinical implications. Crit Care 20:129

2. Miller, J. D., Stanek A, Langfitt TW (1972) Concepts of cerebral perfusion pressure and vascular compression during intracranial hypertension. Prog Brain Res 35:411-432

3. Lassen N (1959) Cerebral blood flow and oxygen consumption in man. Physiol Rev 39:183238

4. Donnelly J, Czosnyka M, Harland S, Varsos G V., Cardim D, Robba C, Liu X, Ainslie PN, Smielewski P (2016) Cerebral haemodynamics during experimental intracranial hypertension. J Cereb Blood Flow Metab. doi: 10.1177/0271678X16639060

5. Harland S, Richards HK, Czosnyka M, Piechnik SK, Pickard JD (1999) Dissociation of cerebral autoregulation and $\mathrm{CO} 2$ reactivity following carotid occlusion in rabbits. J Cereb Blood Flow Metab 19:S636

6. Robba C, Donnelly J, Bertuetti R, Cardim D, Sekhon MS, Aries M, Smielewski P, Richards H, Czosnyka M (2015) Doppler Non-invasive Monitoring of ICP in an Animal Model of Acute Intracranial Hypertension. Neurocrit Care 23:419-426

7. Varsos G V, Richards H, Kasprowicz M, Budohoski KP, Brady KM, Reinhard M, Avolio A, Smielewski P, Pickard JD, Czosnyka M (2013) Critical closing pressure determined with a model of cerebrovascular impedance. J Cereb Blood Flow Metab 33:235-43

8. Dewey RC, Pieper HP, Hunt WE (1974) Experimental cerebral hemodynamics. Vasomotor tone, critical closing pressure, and vascular bed resistance. J Neurosurg 41:597-606

9. Varsos G V., Czosnyka M, Smielewski P, Garnett MR, Liu X, Kim D-J, Donnelly J, Adams H, Pickard JD, Czosnyka Z (2015) Cerebral critical closing pressure in hydrocephalus patients undertaking infusion tests. Neurol. Res. 
10. Horsfield MA, Jara JL, Saeed NP, Panerai RB, Robinson TG (2013) Regional differences in dynamic cerebral autoregulation in the healthy brain assessed by magnetic resonance imaging. PLoS One 8:e62588

11. Zweifel C, Czosnyka M, Lavinio A, Castellani G, Kim D-J, Carrera E, Pickard JD, Kirkpatrick PJ, Smielewski P (2010) A comparison study of cerebral autoregulation assessed with transcranial Doppler and cortical laser Doppler flowmetry. Neurol Res 32:425-428 